IMECE2006-14607

\title{
DYNAMIC SIMULATION OF TRAIN DERAILMENTS
}

\author{
C.R. Paetsch \\ TIAX LLC \\ Cambridge, Massachusetts, USA \\ paetsch.chris@tiaxllc.com
}

\author{
A.B. Perlman \\ Tufts University \\ Medford, Massachusetts, USA \\ bperlman@tufts.edu
}

\author{
D.Y. Jeong \\ U.S. Department of Transportation \\ Volpe National Transportation Systems Center \\ Cambridge, Massachusetts, USA \\ jeong@volpe.dot.gov
}

\begin{abstract}
This paper describes a planar rigid-body model to examine the gross motions of rail cars in a train derailment. The model is implemented using a commercial software package called ADAMS (Automatic Dynamic Analysis of Mechanical Systems). The results of the ADAMS model are compared with results from other engineering models that were developed from explicit derivation of the equations of motion. The ADAMS model was also used to conduct sensitivity studies. Various assumptions and characteristic values were varied to examine their respective effect on the resulting motion. The variations include: the number of cars in the train make-up, onand off-track coefficients of friction, coupler characteristics, and initial conditions. Results from the simulations suggest that train speed, on- and off-track coefficients of friction, and coupler characteristics have the most significant influence on the gross motions.
\end{abstract}

\section{INTRODUCTION}

The need to develop physics-based models to assess the performance of railroad tank cars under accident loading conditions has been identified in the past [1]. Moreover, the need for this research has been highlighted by recent train derailments that led to the accidental release of hazardous materials, loss of life, and property and environmental damage.

- On January 18,2002 , a freight train traveling about 41 miles per hour (mph) derailed near Minot, North Dakota. Five tank cars carrying anhydrous ammonia failed catastrophically, which resulted in one fatality, evacuation of the nearby town, property damage exceeding \$2 million, and over \$8 million on environmental remediation [2].
- On June 28, 2004, a freight train traveling about 45 miles per hour struck the side of a car in another freight train that was entering a siding while traveling about $20 \mathrm{mph}$ in the opposite direction. The collision occurred near Macdona, Texas and resulted in a total of 39 derailed cars, breach of a tank car containing chlorine, three fatalities, and total (i.e., property and environmental) damages exceeding \$7 million [3].

- On January 6, 2005, a freight train traveling about 47 $\mathrm{mph}$ through Graniteville, South Carolina encountered an improperly lined switch that diverted the train from the mainline onto a side track where it collided with an unoccupied, parked train. Among the derailed freight cars were three tank cars, one of which was breached, releasing chlorine. This accident resulted in nine fatalities; evacuation of about 5,400 people, and total damages exceeding $\$ 6.9$ million [4].

Sponsored by the Federal Railroad Administration, the Volpe National Transportation Systems Center (Volpe Center) is conducting and managing a research program to examine the structural integrity of railroad tank cars. This research program comprises three phases to assess the consequences of railroad tank cars involved in derailments. Each phase involves the development of computational models with different objectives: (1) kinematic modeling to estimate the gross motions of rail cars in a train derailment, (2) structural finite element modeling to calculate impact forces inferred from the gross motions, and (3) damage assessment modeling to estimate the deformations of the tank car before catastrophic failure occurs.

This paper describes an engineering model that was developed to guide the first phase of this research program. 
This model was developed using a commercial general purpose software program for rigid multi-body dynamics called ADAMS (Automatic Dynamic Analysis of Mechanical Systems). The model was used to perform sensitivity studies to examine the relative effect of different operational factors on the severity of a train derailment. Different measures were considered to examine derailment severity, such as the number of derailed cars, peak impact forces, and maximum lateral displacement of the derailed cars.

\section{REVIEW OF PREVIOUS WORK}

Previous work in rail accident research can be roughly divided into two categories: (1) investigations of vehicle-track interaction and (2) studies of the actions between cars.

The objective of research in the first category is to understand the mechanisms of derailments (e.g., wheel climb and excessive lateral-to-vertical wheel load ratio). The results from vehicle-track interaction models are not directly applicable to the present work, but they can provide some information regarding modeling techniques.

Research in the second category is directly applicable to the present work, and has been conducted through two approaches: (a) development of special purpose models and (b) development of models using commercial general purpose software for multi-body dynamics.

A special purpose model was developed in the 1970s to examine the gross motions of rail cars in a train derailment [5]. Each car in this planar (i.e., two-dimensional) analysis was assumed to behave as a rigid body of finite length but zero width. A major limitation of this model was that the cars were assumed to remain coupled during the derailment. Another special purpose model was later developed under the sponsorship of Transport Canada (TC) that allowed the cars to decouple, but the criterion for decoupling was incomplete [6,7]. The Transport Canada model included provisions for: tangent or curved track, initial derailment at either the lead or trailing truck of the first car off the track, and potential for tank car rollover based on a vertical-to-horizontal force ratio criterion. More recently, a planar model was developed at Queen's University [8], in which the differential equations of motion were derived from Lagrange's equations and were solved numerically using a Fortran computer program.

Commercial general purpose software for multi-body dynamics has been used to develop a three-dimensional model to examine train crashes [9]. Specifically, a software package called DADS (Dynamic Analysis Design Simulation) was used to calculate the gross motions for a 20 -car train.

\section{ADAMS MODEL}

Rail cars are constructed in ADAMS as a cylindrical part with an arbitrary radius of nine feet. The length of the cylinder depends on whether it represents a tank car or a locomotive. The locomotive is approximately 60 feet, and tank cars are slightly longer at about 65 feet. A planar constraint, connecting the car center of mass to the ground, is used to limit the car's motion to the x-z plane. A planar constraint is only required on one car in a train consist as constraints in the couplers limit the motion of subsequent cars.

The entire train consist is given an initial velocity in the transverse direction and the derailment is modeled with an initial angular velocity on the lead car (with an associated lateral velocity). The car's motion is resisted by a frictional force applied at the car's trucks, which increases as the truck passes the point of derailment.

A basic description of a generic car in the consist and the equations of motion used in the model are presented in the Appendix. Additional details of the ADAMS model are described in [10].

\section{PARAMETRIC STUDIES}

The ADAMS model for derailment kinematics was used to perform sensitivity studies. Baseline parameters were developed and varied one factor at a time to examine their relative effect on (1) the number of derailed cars, (2) the peak and average coupler impact force and restoring torque, and (3) the maximum lateral displacement of a derailed car. The baseline parameters are listed in Table 1 .

\begin{tabular}{|c|c|c|}
\hline Parameter & Baseline Value & Units \\
\hline Cars in Consist & 30 & \\
\hline Lumped Mass Simplification & 0 & \\
\hline Cars with Coupler Breakage & 0 & \\
\hline Cars with Contact Force & 0 & \\
\hline Coupler Length & 4 & feet \\
\hline Coupler Dead Band & 0.25 & feet \\
\hline Maximum Swing Angle & 17.5 & deg \\
\hline Coefficient of Friction - Off & 0.5 & \\
\hline Coefficient of Friction - On & 0.15 & \\
\hline Initial Angular Velocity & 11.46 & deg/sec \\
\hline Initial Longitudinal Velocity & 40 & $\mathrm{mph}$ \\
\hline Coupler Stiffness & $50 \times 10^{6}$ & $\mathrm{lbs} / \mathrm{ft}$ \\
\hline Torque Slope & $1.3 \times 10^{5}$ & lbs-ft/deg \\
\hline Torque Order & 8 & \\
\hline
\end{tabular}

Table 1. Baseline Parameters

The coupler length defines the neutral distance between tank cars. The coupler dead band is the distance the coupler length can displace before a restoring force is applied. The maximum swing angle is the angle at which the restoring moment begins to be applied. The coefficient of friction - on and -off are the values used for the coefficient of friction, $\mu$, in the truck to rail/ground force. The initial angular velocity is the speed at which the lead car is rotating at the beginning of the simulation and the initial longitudinal velocity is the speed at which each car is traveling down the track at the beginning of the simulation. The coupler stiffness is the slope of the coupler force-displacement curve. The torque slope is the coefficient of the torque coupler angle curve. The order is the power of the polynomial term in the torque equation. 
In general, the motion of the train consist begins with an initial rotation imparted to the lead car and with all cars traveling at a given initial speed. The lead car swings out in the direction of the initial rotation, with higher off-track frictional forces applied at the trucks opposing the direction of motion. As the cars derail, the coupler forces between cars create a moment couple. The many highly coupled interactions cause an irregular buckling pattern which generally occurs in two forms, either as an alternating pile-up as shown in Figure 1, or as a curved string of displaced cars as shown in Figure 2.

The direction of travel is left to right in all the figures.

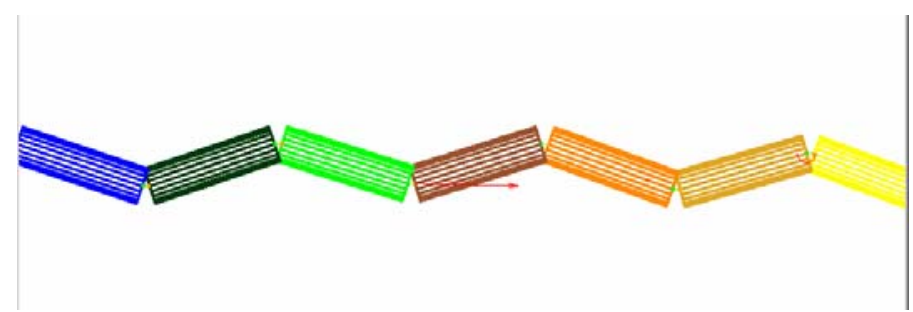

Figure 1: Alternating direction-buckling pattern.

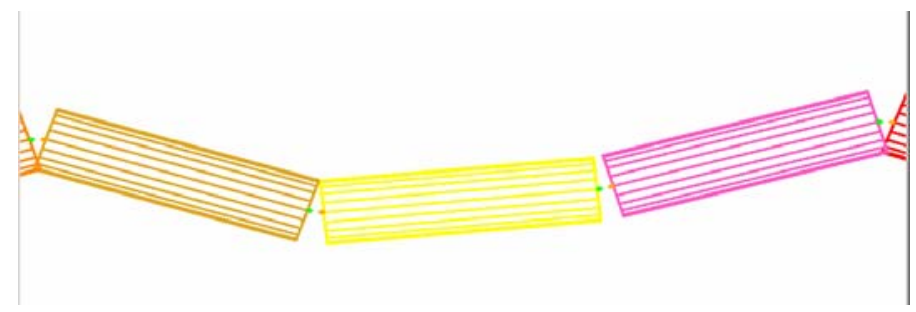

Figure 2: Curved string of displaced cars.

\section{Effect of Train Length}

Derailments occur with train make-ups of varying numbers of cars. Therefore, it is important to understand how the number of cars in the train make-up affects the outcome. The buckling of cars does not necessarily propagate through the entire train consist. Because the pile-up occurs relatively slowly, the rate at which energy is dissipated from the system by the frictional force appears to limit the number of cars derailed. By varying the number of cars in the model, the amount of energy initially in the system is increased, and correlates with the number of cars derailed, as shown in Figure 3. Also, the larger number of cars at the rear of the train tends to create more compression between cars. Correspondingly, the average coupler forces for the first 20 cars increase with the number of cars in the model, as shown in Figure 4.

The maximum force varies less than $20 \%$ over the range of consist size. Even small consists may experience large forces. The location of the maximum force tends to be toward the front, consistently occurring to the rear of the fifth coupler, whereas the maximum moment tended to be located in the first cars, always located in the first 5 couplers. The corresponding average and maximum torque indicates less of a trend with the number of cars in the train consist than the average force, but do generally increase with a greater number of cars. The final comparison criterion, the maximum displacement of the cars, does not vary with the number of cars in the train consist. This observation suggests that other factors must play a part in cars getting off the tracks.

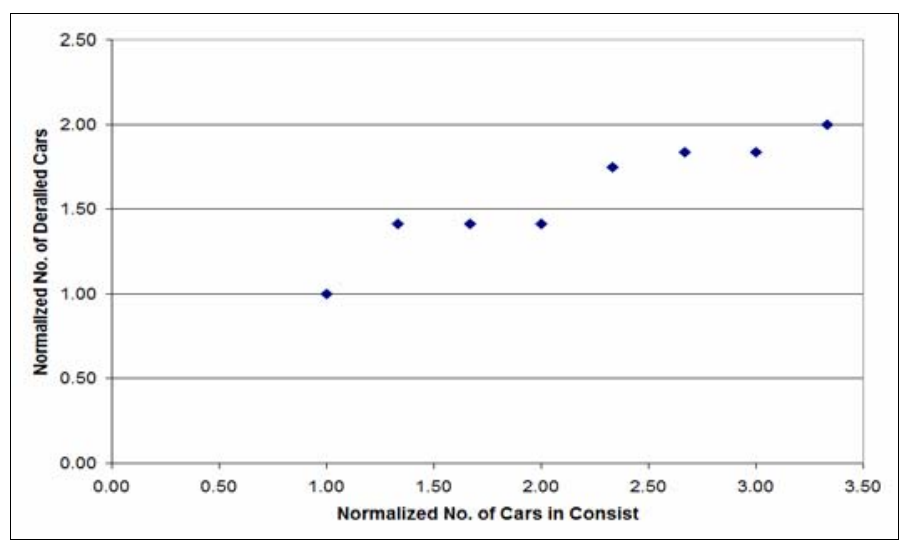

Figure 3: Normalized number of derailed cars for varying number of cars in the consist, normalized about a 30-car model and 12 derailed cars.

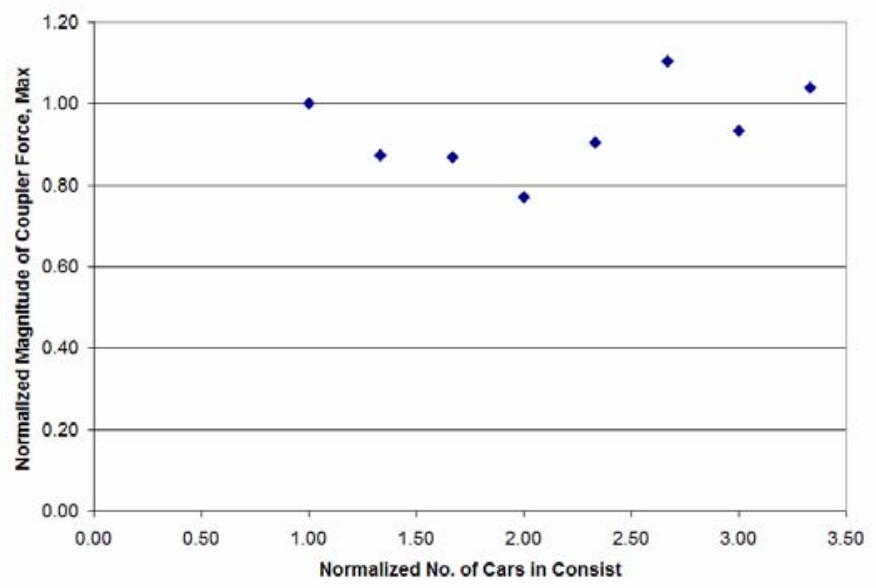

Figure 4: Normalized maximum coupler force for varying number of cars in the consist, normalized about a 30-car model and 7.56 million pounds of force.

Not as easily conveyed in the comparison plots is the large variation in the pile-up pattern of the various models. The specific motion in each model indicates high sensitivity to the number of cars in the train consist, even varying with the addition of a single car. While there may be many factors at play, it does appear that the sensitivity is related to the angular position of the coupler when compressed. As the coupler tends to alternate direction between positive and negative angles frequently, when additional cars change the timing of the coupler forces, a coupler aligned in a different direction can set off an altered pile-up pattern. 


\section{Effect of Train Speed}

Not surprisingly, the initial velocity of the train consist greatly influences the motion of the cars, the derailment characteristics, and the impact forces. At reduced speed, the train is rapidly slowed by the frictional force and the cars come to a quick stop, limiting the distance the lead car is displaced from the track in the z-direction. As the initial speed increases, the cars are in motion for a greater period of time, allowing them to get further from the track. Figure 5 shows that increasing the initial train speed correlates to an increase in the derailment severity in terms of the number of derailed cars.

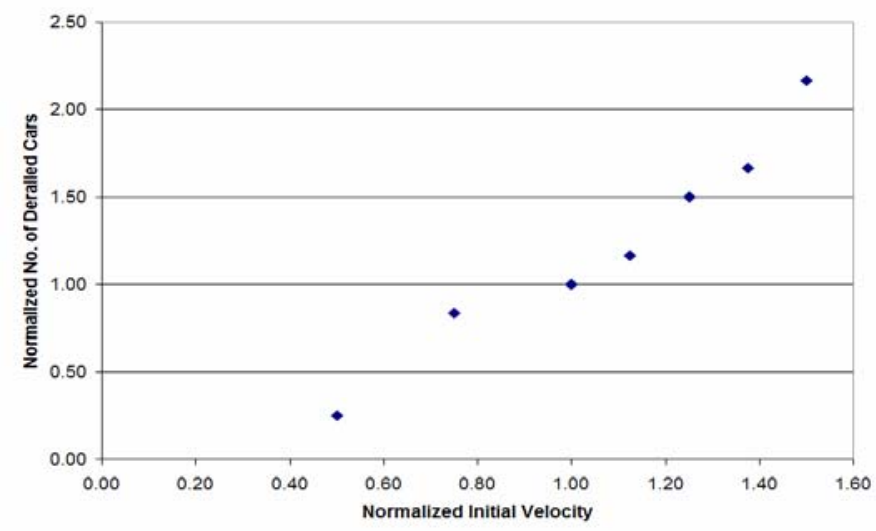

Figure 5: Number of derailed cars for 30-car model with varied initial train speed, normalized about a speed of $\mathbf{4 0}$ miles per hour and 12 derailed cars.

Greater initial speed also displaces cars a greater distance from the track, with up to a $50 \%$ increase in maximum displacement for 1.5 times the initial speed. Additionally, greater initial velocity correlates with an increase in the average and maximum force and torque. When the initial speed is increased by $50 \%$, the average coupler force increases more than 2.5 times the baseline value, as shown in Figure 6.

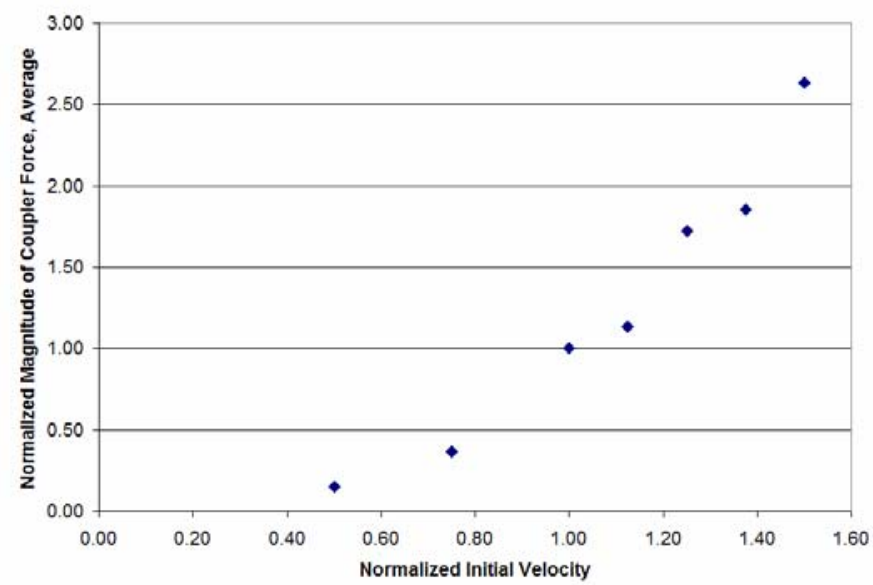

Figure 6: Average coupler force for 30-car model with varied initial train speed, normalized about a speed of $\mathbf{4 0}$ miles per hour and an average force of $1.22 \cdot 10^{5} \mathrm{lbs}$.

\section{Effect of Friction}

The model assumes two types of friction: (1) on-track coefficient of friction is an approximation of the maximum frictional force associated with emergency braking and (2) offtrack coefficient of friction is an estimate of the complicated resistive forces present when the cars derail.

Increasing the off-track friction increases the total dissipative force on the cars that have passed the point of derailment, and the maximum displacement decreases. Additionally, increasing the off-track friction slows the lead cars at a greater rate than the cars not passed the point of derailment. The greater difference in speeds provides more impact energy and a larger number of cars are derailed, with $60 \%$ more cars derailing for twice the friction forces. Figure 7 shows this trend.

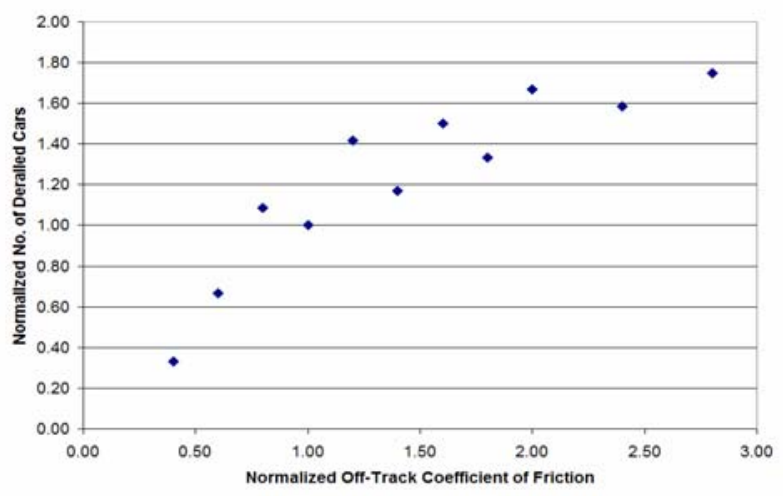

Figure 7: Number of derailed cars for 30-car model with varied off-track coefficient of friction, normalized about a coefficient of 0.5 and 12 derailed cars.

The increase in energy with a larger off-track coefficient of friction also affects the coupler action. With a greater speed differential between lead and rear cars, the restoring forces in the coupler increase and correlations can be seen in the maximum and average force and torque plots, as shown in Figure 8.

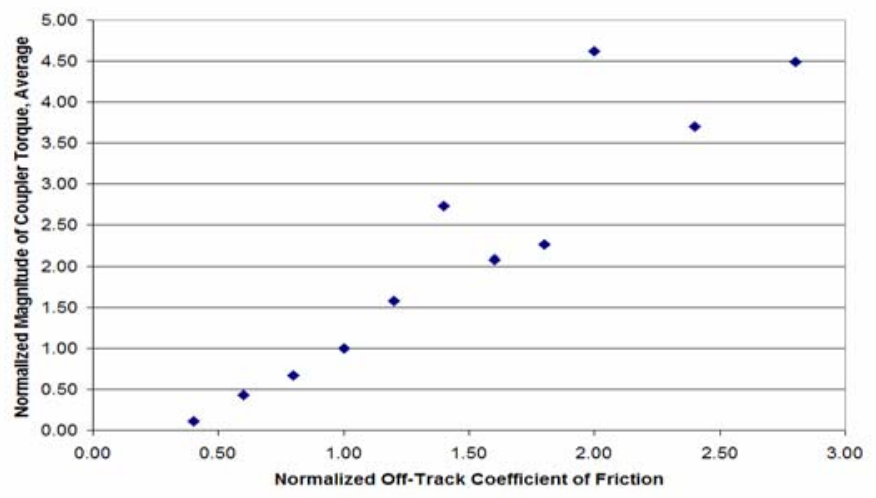

Figure 8: Average coupler torque for 30-car model with varied off-track coefficient of friction, normalized about a coefficient of 0.5 and a average torque of $2.92 \cdot 10^{5} \mathrm{lbs} \cdot \mathrm{ft}$. 
Most of the models tend to develop long, curved strings of cars behind alternating buckling pattern in the lead cars, as shown Figure 9. However, models with 1.4 and 2.0 times the baseline coefficient of friction are outliers with significantly higher average torques. In these two cases, all derailed cars form a buckling pattern of cars in alternating directions, as shown Figure 10. As Figure 8 indicates, derailed cars in alternating directions generally tend to require a larger restoring torque, as maximum swing angles are reached on all couplers. This behavior contrasts with the long, curved strings of cars whose couplers do not reach the maximum swing angle as frequently. The cause of the different buckling patterns is not obvious. Curved strings of cars appear to be associated with higher coupler torque.

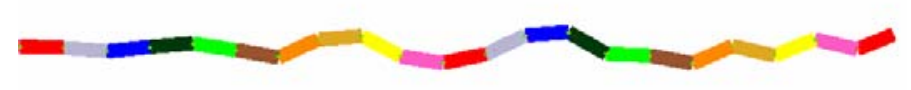

Figure 9: Simulation of 30-car model with an off-track coefficient of friction of 1.4 at 13 seconds.

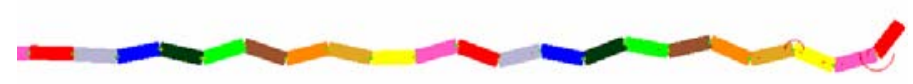

\section{Figure 10: Simulation of 30-car model with an off-track coefficient of friction of 1.0 at 13 seconds.}

Similar to the off-track friction, variation of the on-track coefficient of friction results in substantial differences in the motion of the 30-car model. Consequently, the maximum displacement from the tracks in the z-direction varies no more than $20 \%$ over the range of values simulated.

The 30-car model exhibits much larger sensitivity as the rear cars never pass the point of derailment. By decreasing the on-track frictional force, the rear cars maintain a higher relative velocity. Therefore, the closing speed between off-track and on-track cars increases, resulting in more impact energy. All of the criteria for comparison indicate a correlation between on-track coefficient of friction and the severity of the derailment. Lowering the baseline coefficient of friction by $40 \%$ doubles the number of derailed cars, average torques are nearly eight times greater than the baseline, and average forces increase threefold, as seen in Figure 11. Conversely, increasing the on-track friction decreases the closing velocities, and the derailment is less severe.

Additionally, the variation of the on-track coefficient of friction influences the buckling pattern. With high on-track coefficients of friction, the model tends to derail with a mix of cars buckling in alternating directions and strings of cars curved in the same direction. As the coefficient of friction is increased, the train consist tends to buckle in long strands of curved cars.

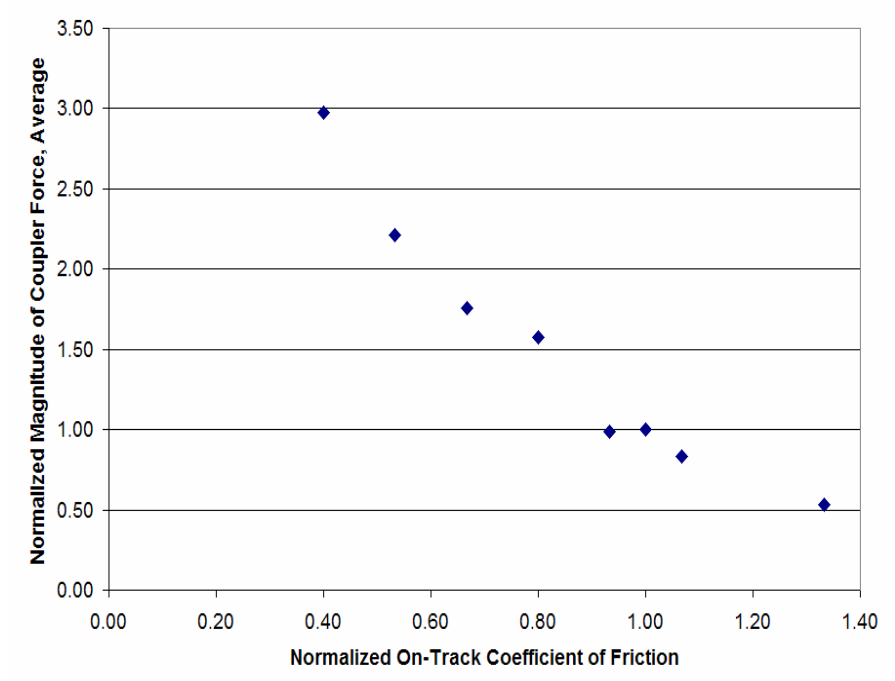

Figure 11: Normalized average coupler force for 30-car models with varied on-track coefficient of friction, normalized about a coefficient of 0.15 .

\section{Effect of Coupler Characteristics}

\section{Coupler length}

The coupler length is altered by initially placing cars the necessary distance apart and adjusting the neutral distance of the coupler in the force equation.

The extension and reduction of the coupler length results in little variation and the comparison criteria do not indicate any trends. The number of derailed cars does tend to increase with coupler length; however the change is not substantial. The maximum and average forces and torques also do not vary for the 30-car model. However, the coupler length does change the overall motion of the cars, as the longer couplers tend to create a gentler buckling pattern in the cars. With a coupler length shorter than the baseline model, the first 10 derailed cars buckle in alternating directions, as shown in Figure 12.

\section{Figure 12: Alternating buckling pattern with a short coupler length.}

However, the longer couplers cause the cars to derail with a smaller angular displacement in the lead cars and trailing cars form gently curved strings of cars, as shown in Figure 13. 


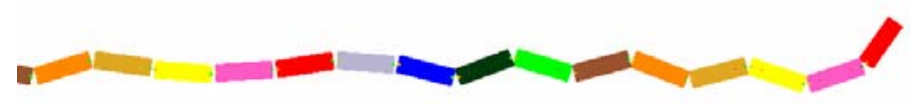

Figure 13: Alternating buckling in the lead cars, gently curved strings trailing with longer coupler.

Coupler length plays an important part in the direction of the applied force, which is only apparent in the graphical representations of the motion. The ability to recognize this difference in motion is a result of ADAMS ability to output a graphical representation of the resulting motion and proves to be a useful tool.

\section{Coupler Dead Band}

The correlation between coupler dead band, representing longitudinal play in the coupler, and the average force is related to the increase in frequency of coupler bottoming. With little play in the coupler, the restoring force is engaged more often.

While the larger dead band has a much larger maximum force, the majority of time coupler force is not applied. With no dead band, the coupler force is always applied so that a smaller maximum force is needed to restore the coupler length. Therefore the dead band average force is approximately $75 \%$ of the model with no dead band. With smaller dead bands, the average forces are higher. Figure 14 indicates over twice the average force for $20 \%$ of the baseline dead band length.

Despite the strong correlation and large variance in the average force, there is not a strong sensitivity to the coupler dead band. It should be noted that the increased interaction in the smaller dead band models require significantly more time to solve.

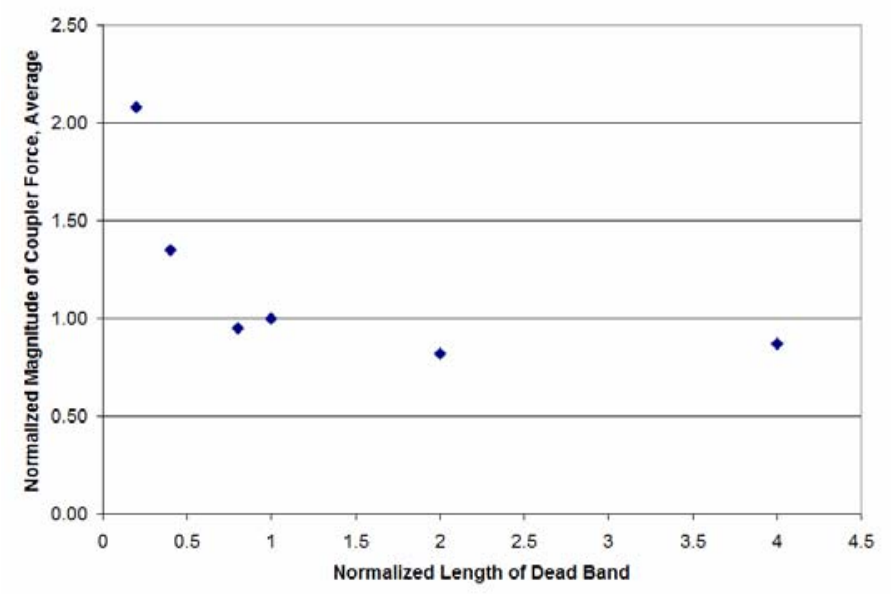

Figure 14: Average coupler force for 30-car models with varied dead bands, normalized about a dead band of 0.25 feet and an average force of $1.22 \cdot 10^{5} \mathrm{lbs}$.

\section{Maximum Swing Angle}

With the variation of the maximum swing angle, the general motion remains similar to the baseline 30-car model. However, with more restricted coupler motion, the pile-up characteristics are altered. As the maximum swing angle becomes smaller, there is little alternating buckling pattern. The majority of buckling occurs in longer, curved strands of cars as shown in Figure 15. As the maximum swing angle is increased beyond the baseline value, the buckling pattern becomes purely alternating, as shown in Figure 16.

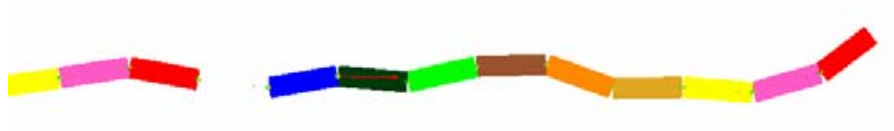

Figure 15: Pile-up pattern of 30-car model with a maximum swing angle of 10 degrees.

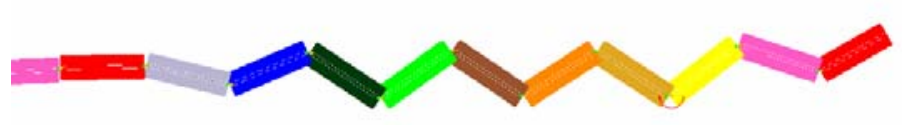

Figure 16: Pile-up pattern of 30-car model with a maximum swing angle of 35 degrees.

The variation in the maximum swing angle indicates the larger angles, with alternating buckling patterns, tend to derail fewer cars. However, the change is not significant, varying only $20 \%$ over the range of swing angles, as shown in Figure 17.

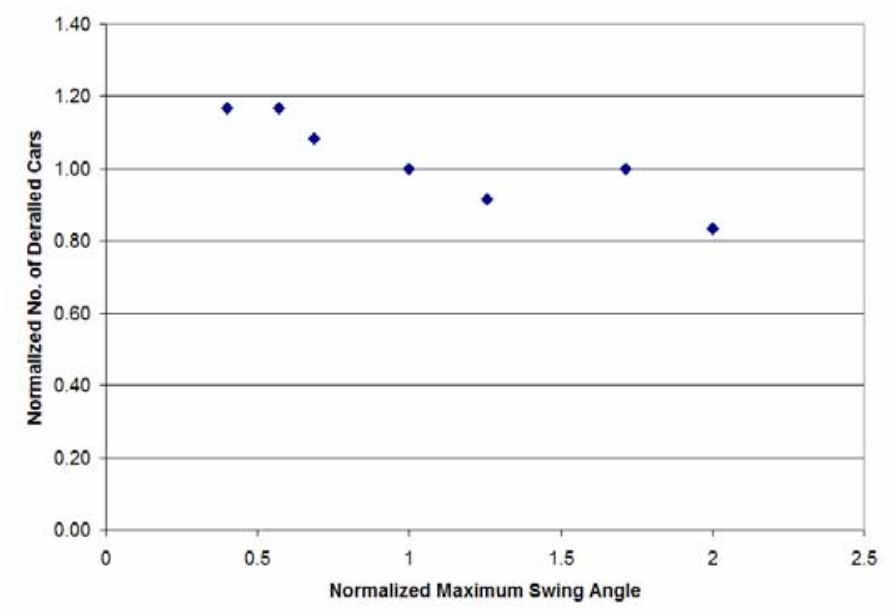

Figure 17: Number of derailed cars for 30-car models with varied maximum swing angles, normalized about an angle of 17.5 degrees and associated 12 derailed cars. 


\section{Coupler Stiffness, Torque Slope, and Torque Order}

The three parameters defining the value of the general force and torque equations, the coupler stiffness, torque slope, and torque order, all had similar results. Therefore, the following outlines the sensitivity of these parameters together.

None of the three parameters exhibited any influence over the motion of the train consist. Consequently, there is little variation in the number of derailed cars or the maximum displacement from the track. However, the maximum force varies with the coupler stiffness and the maximum torque varies with the torque slope and order. For example, Figure 18 illustrates the correlation between the maximum coupler force and coupler stiffness. Yet, the corresponding average force does not vary, as shown in Figure 19.

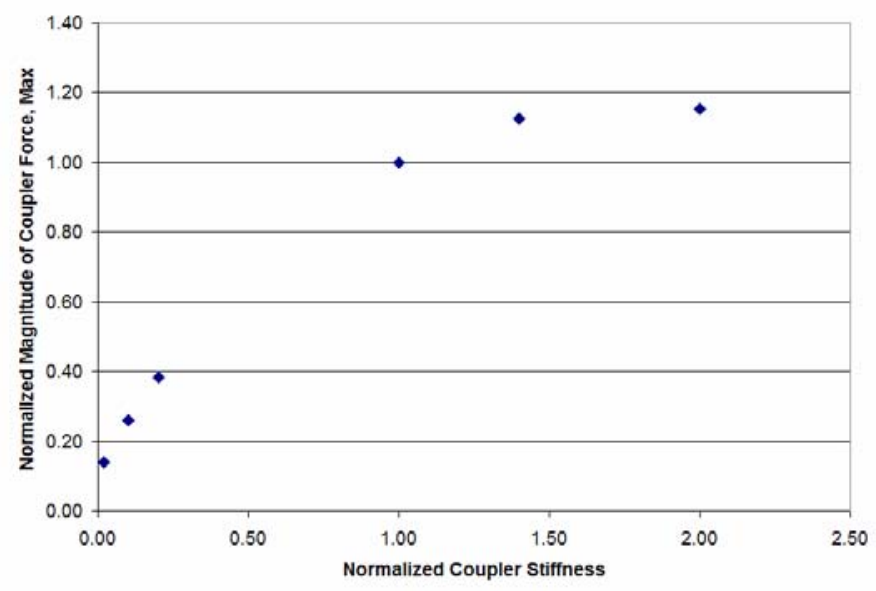

Figure 18: Maximum coupler force for 30-car model with varied coupler stiffness, normalized about a stiffness of 50 . $10^{6} \mathrm{lbs}$ per foot and $7.56 \cdot 10^{6} \mathrm{lbs}$.

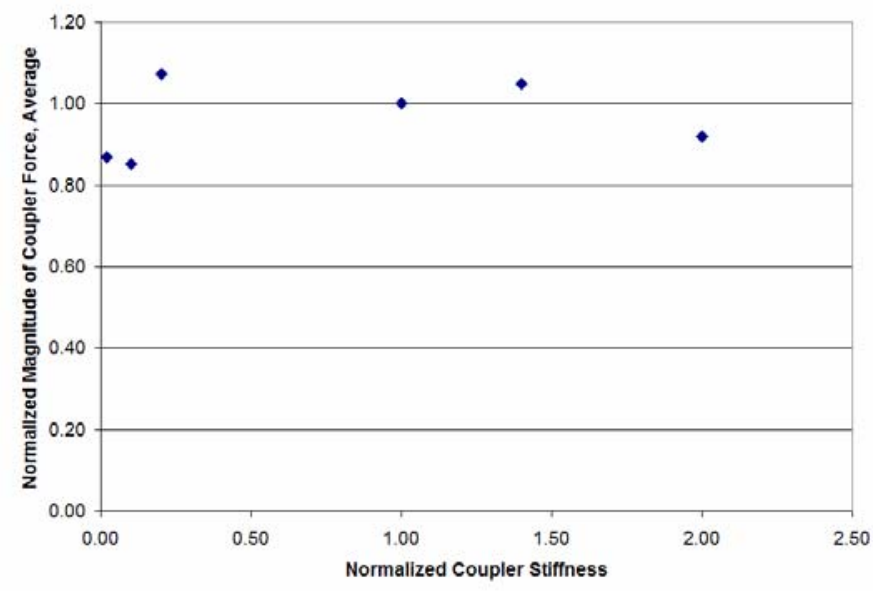

Figure 19: Normalized average coupler force for 30 -car model with varied coupler stiffness, normalized about a stiffness of $50 \cdot 10^{6} \mathrm{lb} / \mathrm{ft}$.

\section{MAXIMUM CLOSING SPEEDS AND IMPACT FORCES}

The parameter tests indicate that specific model values can affect the system's dynamics. However, the model is not very sensitive to these variations. Therefore, the closing velocities and maximum forces determined from the models provide an approximation of derailment dynamics, bounding the range of values.

In order to determine the closing velocities and maximum forces, two models were used. First, to account for the importance of the number of cars in the consist, a 100-car model was used to incorporate the dynamics of a long consist. A second 30-car model with 70 cars of equivalent mass, was used to evaluate the effects of coupler breaks and contact forces for the first 20 cars.

Figure 20 illustrates the basic pattern for a 100-car model derailment. The lead car initiates the derailment. As the car steers off the tracks and passes the point of the derailment, it begins to slow at a greater rate than the trailing cars. The differential in speed causes the second car to collide with the lead car and a coupler force is applied. Behind the lead cars, the buckling pattern is initiated with couplers coming out of line. The consist continues to buckle until the frictional force dissipates the system's energy and the cars come to rest, taking about 13 seconds. A total of 24 cars are derailed in the process.

While the maximum closing velocities may occur with any given distance between the adjacent cars, the largest difference in longitudinal component of velocity generally occurs when the coupler is at its minimum length. When rotation of the vehicles is considered, the maximum generally occurs as the cars reach their maximum buckling amplitude. This model provides an approximation of the impact forces that does not account for coupler break.

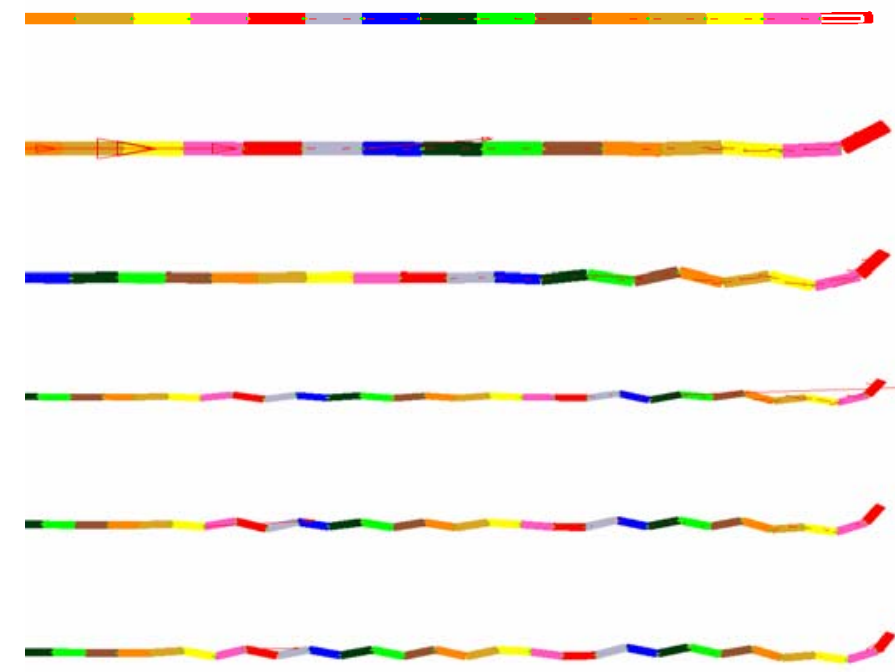

Figure 20: 100-car model derailing, shown at time steps 0.1, $4,5,7,9,11$, and 13 seconds, respectively. 
To capture inter-car action, a consist of 30 -cars with a 70 car equivalent mass lumped into the 30th car was implemented. Coupler break capability and the possibility of contact forces were activated on only the first 10 cars in the consist. Allowing the couplers to break significantly reduces the interaction between the cars. There are two categories of these interactions, initial and secondary contact. Initial interaction, the first contact between pairs of cars, occurs just after the cars decouple. Secondary contact includes all impacts that occur after the cars have been decoupled.

Figure 21 shows a typical derailment pattern for the model when coupler break is included. The evolution of the derailment shows how a pile-up configuration develops.
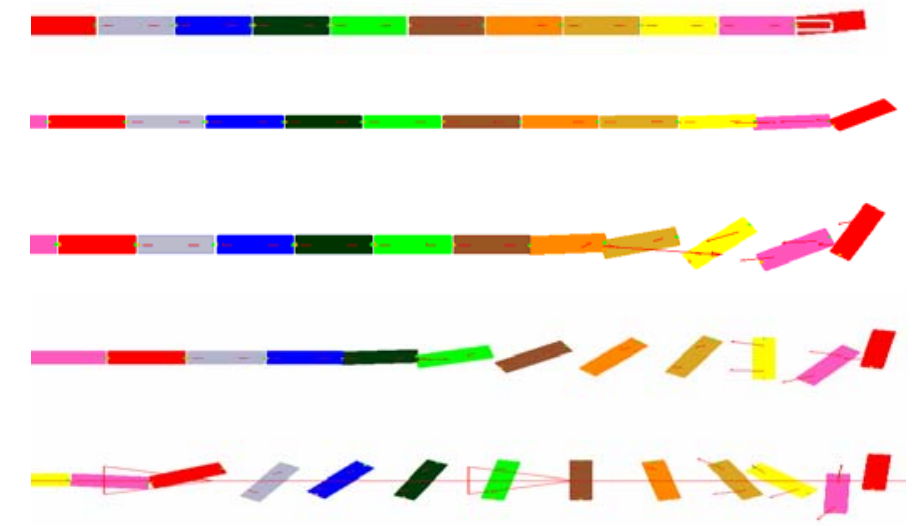

Figure 21: 30-car model with 70 car equivalent lumped mass, and coupler breaks and contact forces applied to the first 10 cars decoupling at $0.75,2.5,3.5,4.75,6.25$ seconds, respectively.

The corresponding closing velocities and impact forces of the initial impacts are shown in Figure 22 and Figure 23.

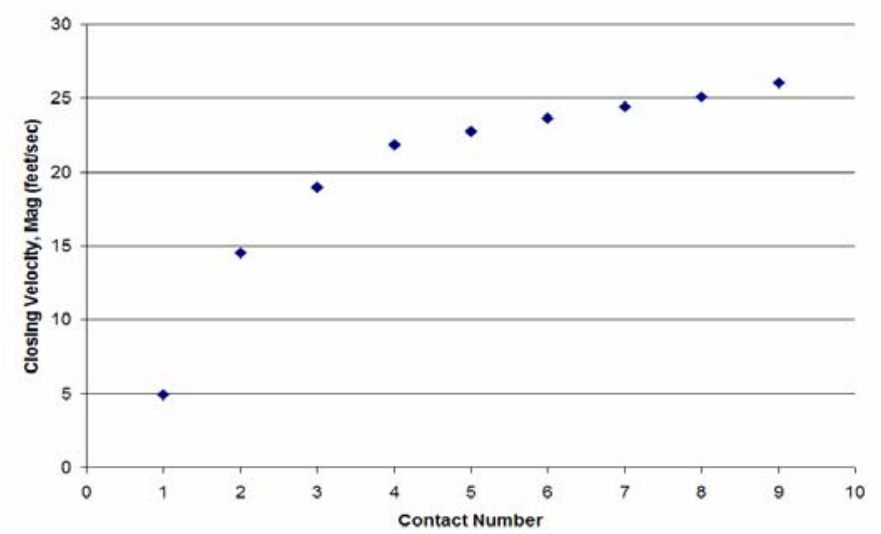

Figure 22: Closing velocities for initial contact in 30-car model with 70-car equivalent lumped mass

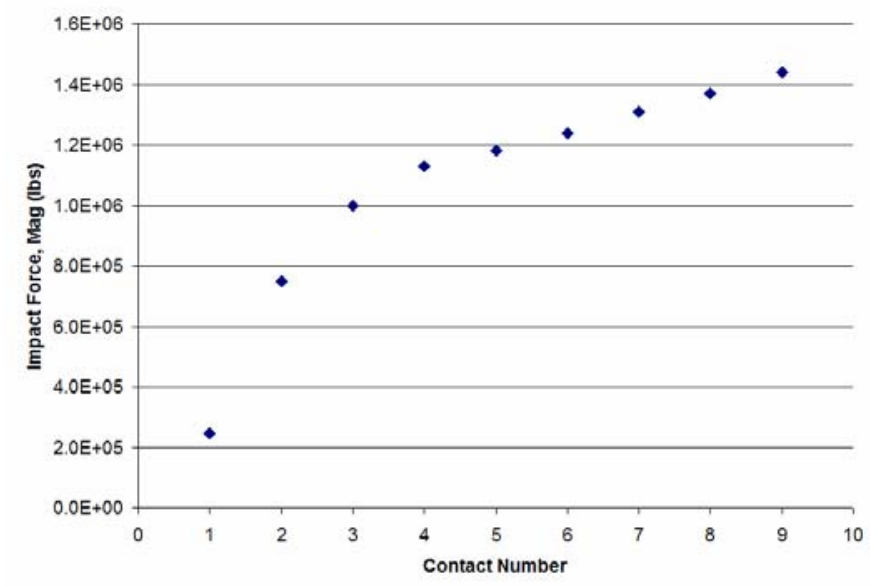

Figure 23: Impact forces for initial contact in 30-car model with 70 car equivalent lumped mass

The action of the secondary contact is illustrated in Figure 24. Each event is captured in the figures below. The cars remain in order corresponding to their original position in the consist.

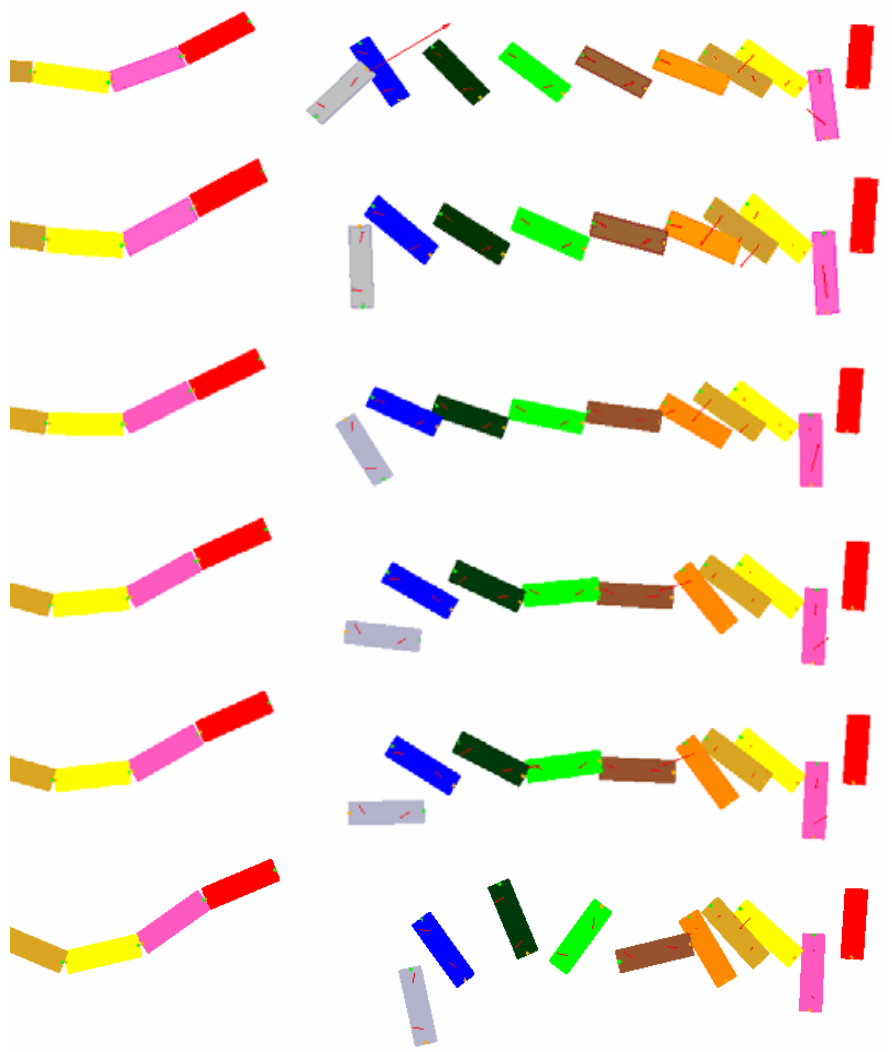

Figure 24: 30-car model with 70 car equivalent lumped mass, and coupler breaks and contact forces applied to the first 10 cars. 
The closing velocities for the secondary contacts are given in Figure 25.

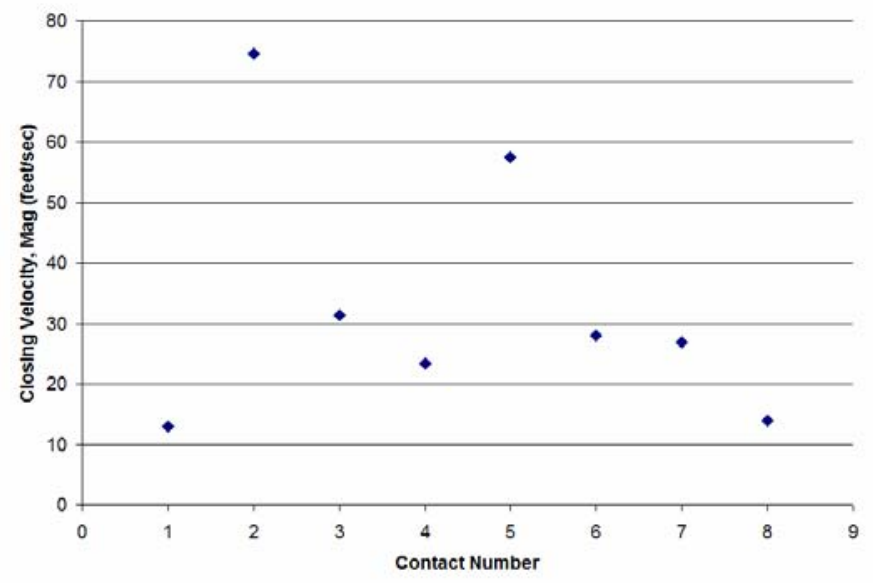

Figure 25: Closing velocities for secondary contact in a 30car model with 70 car equivalent mass

The secondary contact results in a wide range of closing velocities, with a minimum of 13 feet per second and a maximum of 75 feet per second. As the initial contact involved relatively little differential in angular velocity, it appears that the secondary contacts' generally larger differentials in angular velocities result in the larger closing velocities. The large closing speed of Contact 2 is a result of both the difference in translational and rotational velocities of Cars 9 and 10 . Additionally, the large variance in the secondary contact closing velocities contrasts the general trend found for contact, and follows from the relatively orderly motion of the initial decoupling and the ensuing non-uniform motion of the secondary contacts.

The impact forces also exhibit a large variance amongst the contact events, as shown in Figure 26.

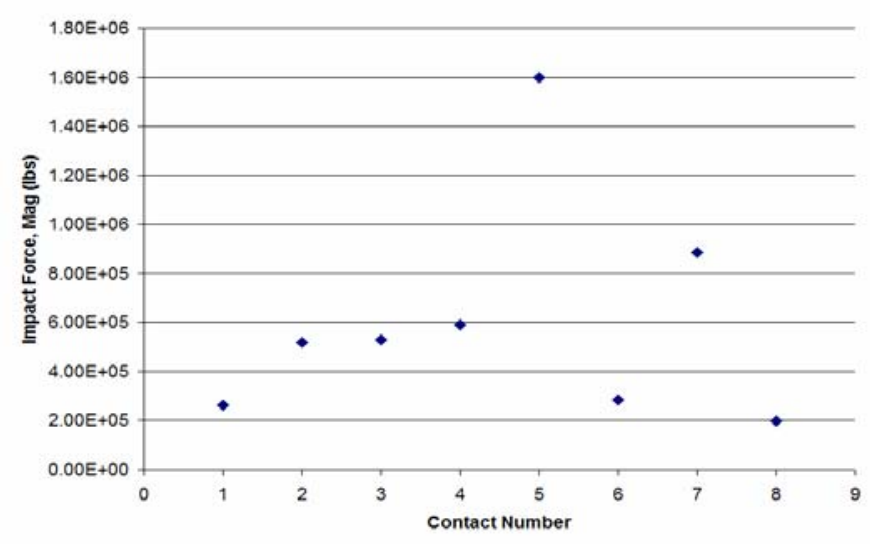

Figure 26: Impact forces for initial contact in 30-car model with 70-car equivalent lumped mass.
Unlike the initial impact forces, the secondary interactions do not correlate with closing velocities and impact forces. The majority of the maximum forces are significantly lower than those for initial impacts. The secondary contact is dominated by a change in the angular momentum, while the initial contacts are mostly in a change of linear momentum.

\section{CONCLUDING REMARKS}

The results of the sensitivity studies indicate that the gross motions of the rail cars in a derailment strongly depend on the number of cars in the train consist, decoupling, and contact forces.

The initial translational and rotational velocities demonstrated significant influence on the derailment severity.

The importance of the initial rotational velocity indicates a strong relationship between the derailment dynamics and the means of derailment.

\section{REFERENCES}

[1] Transportation Research Board, 1994: "Ensuring Tank Car Safety," TRB Special Report 243, Washington, DC.

[2] National Transportation Safety Board, "Derailment of Canadian Pacific Railway Freight Train 292-16 and Subsequent Release of Anhydrous Ammonia Near Minot, North Dakota January 18, 2002," Railroad Accident Report NTSB/RAR-04/01, March 2004.

[3] National Transportation Safety Board, "Collision of Union Pacific Railroad Train MHOTU-23 With BNSF Railway Company Train MEAP-TUL-126-D With Subsequent Derailment and Hazardous Materials Release, Macdona, Texas, June 28, 2004" Railroad Accident Report NTSB/RAR-06/03, July 2006.

[4] National Transportation Safety Board, "Collision of Norfolk Southern Freight Train 192 With Standing Norfolk Southern Local Train P22 With Subsequent Hazardous Materials Release at Graniteville, South Carolina January 6, 2005," Railroad Accident Report NTSB/RAR-05/04, November 2005.

[5] Yang, T.H., Manos, W.B., Johnstone, B., 1972: "Dynamic Analysis of Train Derailments," ASME Winter Annual Meeting, ASME Paper 72-WA/RT-5.

[6] Birk, A.M., Anderson, R.J., Coppens, A.J., 1990: "Computer simulation of a derailment accident, Part I Model basis," Journal of Hazardous Materials 25, 121147.

[7] Birk, A.M., Anderson, R.J., Coppens, A.J., 1990: "Computer simulation of a derailment accident, Part II Sample simulation," Journal of Hazardous Materials 25, 149-165.

[8] Toma, E.E., 1998: "A Computer Model of a Train Derailment,” Ph.D. Thesis, Queen's University, Kingston, Ontario, Canada.

[9] Han, H.S., Koo, J.S., 2003: "Simulation of train crash in three dimensions," Vehicle System Dynamics 20, 435-450. 
[10]Paetsch, C.R., 2005: "Dynamic Simulation of Tank Car Derailments," M.S. Thesis, Tufts University, Medford, Massachusetts, USA.

\section{APPENDIX}

A simplified, two-dimensional model was created to address the needs of the study. Each car is represented as a free body with three degrees of freedom in the horizontal plane, two translational motions and one rotational. Cars have assigned rotational mass moment of inertia for a thin rod.

The truck-to-ground constraints are modeled as frictional forces applied at the truck to car center. It is described as Coulomb friction, dependent on the coefficient of friction, car weight, and direction of motion, and represents the resistance to motion between the car and the track or ground. Each type of interaction, truck-rail or truck-ground, is represented by different coefficients of friction, dependent only on the longitudinal displacement of the truck relative to the point of derailment.

The coupler is comprised of two smaller bodies connected to the end of each car with revolute joints. Additionally, reaction at the coupler is modeled as a spring force and a pair of moments applied to the ends of adjacent cars. All coupler forces and torques are applied to the two bodies and a linear constraint maintains the alignment of the two bodies. A coupler spring force restrains the motion between adjacent cars and models the draft gear along with any other form of end-of-car cushioning device. Therefore, there is a dead band included in the calculation to account for these physical attributes. A moment applied at each end of the coupler represents the coupler impacting the sides of the coupler pocket. This is an approximation to the actual dynamics, as the saturation of the coupler swing would result in lateral force acting on the angled side of the bell mouth. The resistive coupler moment is expressed as a polynomial function of the coupler angle. It includes a dead band when the swing angle is less than the angle of the bell mouth.

A generalized application of these forces and moments to the free bodies is shown in Figure 27.

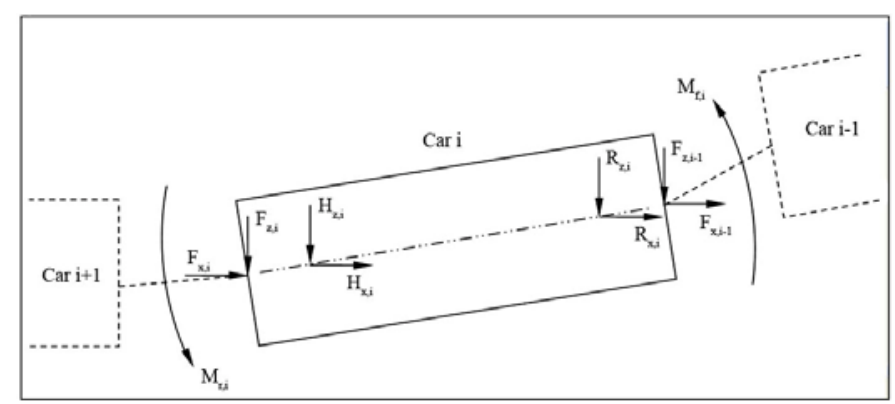

Figure 27: Forces and torques applied to Car i.

The equations of motion assume a reference frame with the $y$ axis normal to the plane of the vehicle. The forces in the $x-$ and $\mathrm{z}$-directions, and the moments are summed below for a generic car. The lead and last cars in the consist have coupler forces at only one end.

$$
\begin{aligned}
& \sum F_{x, i}=R_{x, i}+H_{x, i}-F_{x, i-1}+F_{x, i}-E_{i-1} \sin \left(\beta_{i-1}\right)+E_{i} \sin \left(\beta_{i}\right) \\
& \sum F_{y, i}=R_{y, i}+H_{y, i}-F_{y, i-1}+F_{y, i}-E_{i-1} \cos \left(\beta_{i-1}\right)+E_{i} \cos \left(\beta_{i}\right) \\
& \sum M_{i}=\frac{1}{2} L_{i}\left(F_{x, i-1}^{2}+F_{y, i-1}^{2}\right)^{\frac{1}{2}} \sin \left(\theta_{i}-\beta_{i-1}\right)+\frac{1}{2} L_{i}\left(F_{x, i}^{2}+F_{y, i}^{2}\right)^{\frac{1}{2}} \sin \left(\theta_{i}-\beta_{i-1}\right) \\
& \quad+\frac{1}{2} D_{i}\left[\left(R_{x, i}^{2}+R_{y, i}^{2}\right)^{\frac{1}{2}}+\left(H_{x, i}^{2}+H_{y, i}^{2}\right)^{\frac{1}{2}}\right]\left[\sin \left(\theta_{i}-\alpha_{f, i}\right)-\sin \left(\theta_{i}-\alpha_{r, i}\right)\right] \\
& +M_{f, i}+M_{r, i}+E_{i-1} \cos \left(\theta_{i}-\beta_{i-1}\right)+E_{i} \cos \left(\theta_{i}-\beta_{i}\right)
\end{aligned}
$$

The summed forces and moments are then set equal to the inertial terms to complete the equations of motion for the $\mathrm{i}^{\text {th }}$ car.

When applicable, the breaking of the coupler due to tension, compression, or bending is modeled. A system variable is created to limit the coupler force. If any of the load magnitudes exceed a prescribed maximum value, a coupler's force and torques are set to zero.

Derailment is initiated by applying an initial rotational velocity to the lead car only. The derailment condition is applied to trailing cars by increasing their frictional forces as the trucks pass the point of initial derailment. 